\title{
Hydrodynamic instabilities in ferronematics
}

\author{
A.B. Ryskin, H. Pleiner, and H.-W. Müller \\ Max Planck Institute for Polymer Research, 55021 Mainz, Germany
}

\section{Introduction}

In the hydrodynamic description of ferronematics there are various dynamic magnetic field effects, linear in the field strength, that are negligible in usual nematics, but can play a role in ferronematics. In ordinary nematics those effects are always neglected, but in ferronematics with their strong sensitivity to magnetic fields there is the expectation that these effects are sufficiently enhanced. They can be described as linear-field-dependent additions to ordinary dynamic material tensors describing, for example, heat conduction, diffusion, electric conductivity, viscosity, flow alignment and relaxation of the director. Since a magnetic field is odd under time reversal symmetry, these new effects are reversible (non-dissipative), if the field-free part of the tensor describes a dissipative effect and vice versa. In isotropic systems a few of such effects are known (Hall and Righi-Leduc effect). Here we investigate theoretically the influence of these new terms on the thermal convection (Bénard) and the viscous fingering (Saffman-Taylor) instability in ferronematics in the presence of a strong magnetic field. We find that the instabilities are qualitatively changed due to the occurrence of a finite vorticity component - a feature that is known from ordinary nematics in the case of a superimposed mechanical rotation. We suggest to use the additional effects (crossflow within convection rolls, oblique rolls, rotating fingers) for measuring the phenomenological coefficients involved.

\section{Rayleigh-Bénard instability}

\subsection{The case when $n \| H$}

We consider an infinitely extended layer of ferronematic liquid crystal bounded by two rigid parallel plates at distance $h$. The temperature of the plates is kept fixed at $T_{1}$ and $T_{0}>T_{1}$ (Fig. 1). An

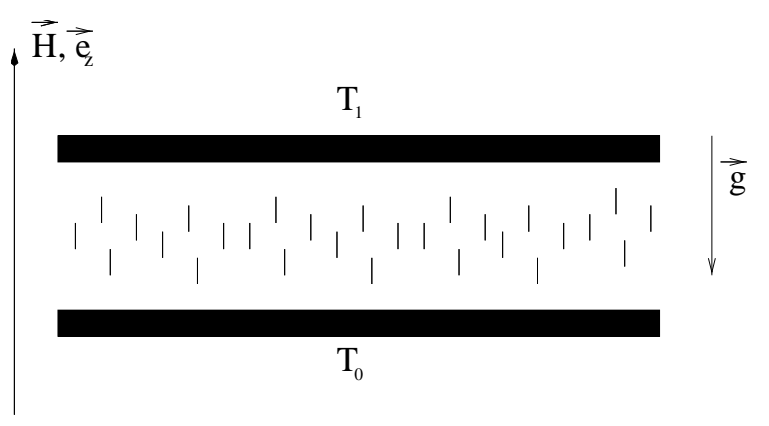

Figure 1: Sketch of the setup in the parallel case. For details see text.

external magnetic field is imposed in $z$-direction $\left(\hat{\boldsymbol{e}}_{z}\right)$ and the gravitational force works in $-z$ direction $\left(\boldsymbol{g}=-g \hat{\boldsymbol{e}}_{z}\right)$. In the case of a positive magnetic susceptibility anisotropy $\chi_{a}>0$ the director tends to align along the magnetic field. Homeotropic boundary conditions for the director are helpful. We assume the magnetic field to be strong enough that the director is clamped and does not have an independent dynamics. The magnetic field in the sample is always taken as static 
and uniform, and equal to the value of external field. Thus the system is described by the effective Navier-Stokes equation, incompressibility and heat conduction.

The trivial heat conduction state, without any flow and a linear temperature profile is always a solution. However, this solution is stable for small temperature differences only and is subject to the Bénard instability, when the temperature difference exceeds some threshold value. To find this threshold value in terms of the material parameters involved, we study the stability of small perturbations of the ground state

It is shown that in addition to those degrees of freedom, which are necessary to describe the Bénard instability in usual nematics, in ferronematics there is also the $z$ - component of the vorticity. This situation is similar to the case of the thermal instability in a rotating layer of simple liquids [2]. In both cases the time reversal symmetry is broken by the external (flow or magnetic) field. In our geometry we expect a roll pattern due to the spatial up-down or midplane symmetry that is still present. In such a pattern the $z$-component of the vorticity is manifest as a crossflow in the $x-y$ plane. Measuring this component of the velocity can serve as a direct indication of the presence of the new field-dependent terms in the viscosity tensor.

\section{$2.2 \quad$ The case when $n \perp H$}

When the magnetic susceptibility anisotropy is negative $\chi_{a}<0$, the director field tends to be perpendicular to the magnetic field.

To consider the influence of the new magnetic field dependent terms we consider the geometry shown in Fig. 2. As in the previous case we have an infinite layer of ferronematics subject to a temperature gradient across the layer. An external magnetic field is imposed along the temperature gradient ( $z$-direction, ) while the nematic director is oriented perpendicular ( $y$-direction). We assume that also a strong electric field is applied, in order to clamp the director in its equilibrium orientation. Thus, like in the preceding section, director reorientations are neglected.

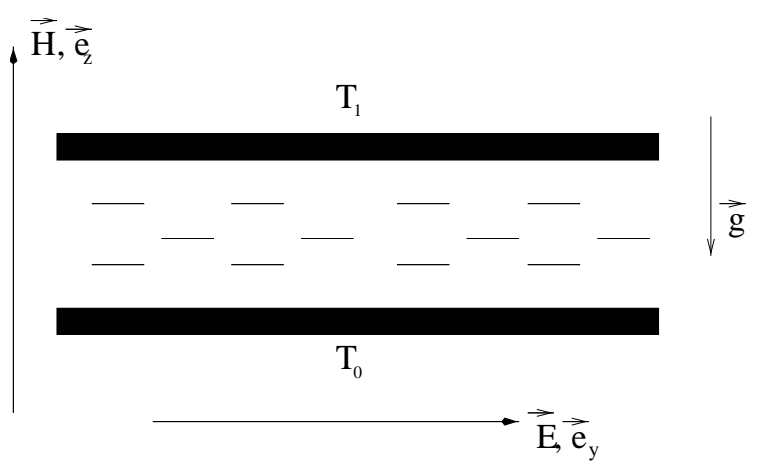

Figure 2: Sketch of the setup in the perpendicular case. For details see text.

To study this problem in more detail we use a three dimensional ansatz introduced in [3] and the solution method presented in Ref. [4].

Above a critical temperature difference that depends on the magnetic field strength convection sets in. For the flow pattern above threshold the following picture emerges. When the magnetic field is absent or small the convective rolls are aligned along the electric field. When the magnetic field exceeds some value $\bar{H}_{\ell}$, the rolls get oblique with respect to the electric field and the angle between the rolls and the electric field increases with increasing magnetic field. At the point when the magnetic field reaches an upper critical value $H_{u}$, the rolls are perpendicular to the electric field and stay so for any higher field. Since the director is always parallel to the electric field (and perpendicular the external magnetic field) it is oblique to the roll orientation in the intermediate magnetic field regime, while in the high field regime it is perpendicular. It is possible that this high field regime cannot be reached in actual experiments. 


\section{Saffman-Taylor instability}

Another useful tool to study the new linear field dependent contributions in the effective viscosity tensor is flow in a Hele-Shaw cell. When a viscous fluid is displaced by a less viscous one in the narrow space of a Hele-Shaw cell, the Saffman-Taylor instability arises [6]. We consider a radial Hele-Shaw cell (Fig. 3), which consists of two parallel transparent plates at a distance $d$. The gap between them is filled with a high viscosity fluid, in our case a ferronematic. The low viscosity one (usually air) is injected through an inlet at the center of the upper plate. A magnetic field is

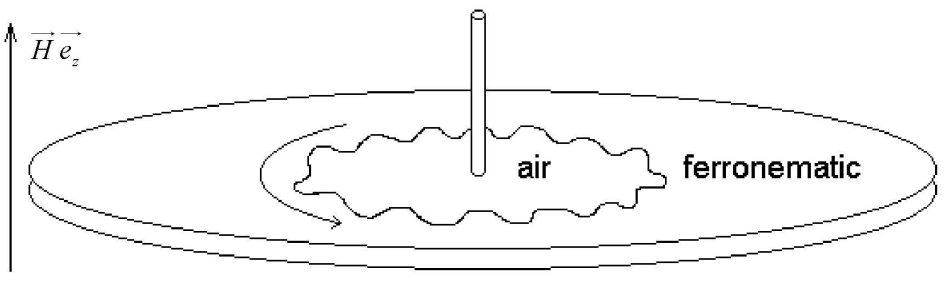

Figure 3: The setup of a radial Hele-Shaw cell. For a ferronematic the external magnetic field leads to the rotation of the fingers shown by the arrow.

imposed perpendicular to the plates (in $z$-direction) strong enough for the nematic director to be clamped. Here we assume that the magnetic susceptibility anisotropy is positive and the director field is aligned parallel to the external field.

There is a trivial solution, when the less viscous liquid extends itself in the form of a circle. It is known, however, that such a solution is unstable against the formation of fingers on the

interface [6]. In the case of ferronematics, in addition to the amplitude amplification of the surface perturbations (finger formation), there is a rotation of the growing fingers with angular velocity proportional to the magnetic field. A field inversion reverses the rotation sense of the fingers.

\section{References}

[1] E. Jarkova, H. Pleiner, H.-W. Müller, A. Fink, and H.R. Brand, Euro. Phys. J. E5 (2001) 583.

[2] S. Chandrasekhar, Hydrodynamic and Hydromagnetic Stability, Clarendon Press, Oxford 1961.

[3] F.G. Busse, J. Fluid Mech. 52/1 (1972) 97.

[4] N. Li, J.O. Murphy, and J.M. Steiner, Z. Angew. Math. Mech. 75 (1995) 3.

[5] A. Buka, Viscous Fingering in Pattern Formation in Liquid Crystals, Springer, New York 1996 and references therein.

[6] P. G. Saffman and G. I. Taylor, Proc. Roy. Soc. London, Ser.A 245 (1958) 312. 